\title{
Malaria Infection is High at Transit and Destination Phases Among Seasonal Migrant Workers in Development Corridors of Northwest Ethiopia: A Repeated Cross-Sectional Study
}

\author{
Tesfaye Tilaye $\mathbb{D}^{\prime}$ \\ Belay Tessema ${ }^{2}$ \\ Kassahun Alemu (iD ${ }^{3}$ \\ 'Institute of Public Health, College of \\ Medicine and Health Sciences, \\ University of Gondar, Gondar, Ethiopia; \\ ${ }^{2}$ Department of Medical Microbiology, \\ School of Biomedical and Medical \\ Laboratory, College of Medicine and \\ Health Sciences, University of Gondar, \\ Gondar, Ethiopia; ${ }^{3}$ Department of \\ Epidemiology and Biostatistics, Institute \\ of Public Health, College of Medicine \\ and Health Sciences, University of \\ Gondar, Gondar, Ethiopia
}

\begin{abstract}
Purpose: Malaria is a leading public health problem in Ethiopia. Every year, thousands of seasonal farm workers travel to farm corridors in Northwest Ethiopia and fall at risk of malaria infection. However, the magnitude of malaria infection and risk factors during harvest time were not well identified. This study aimed at estimating the prevalence and risk factors of malaria infection among seasonal migrant workers in Northwest Ethiopia.

Methods: A repeated cross-sectional study was conducted at transit and destination phases in Metema district from September 15, 2018 to October 30, 2019. Data were collected using a structured questionnaire. A capillary blood sample was collected to examine infection with malaria parasite using a microscope. A multivariate logistic regression technique was used to determine risk factors.

Results: The malaria prevalence at transit and destination phases among migrant workers was $13.5 \%$ (95\% CI: $12.07-14.93 \%$ ) and $18.7 \%$ (95\% CI: $16.40-21.02 \%)$, respectively. The combined prevalence was $16.1 \%$ (95\% CI: $14.67-17.63 \%)$. The odds of malaria infection among migrant workers at the destination phase was $1.5(\mathrm{OR}=1.5,95 \%$ CI $1.167-1.846)$ times higher compared to the transit phase. Education (AOR=8.198; 95\% CI: 4.318-15.564), knowledge of antimalarial drugs $(\mathrm{AOR}=2.4 ; 95 \% \mathrm{CI}: 1.43-3.95)$, and use of long-lasting insecticidal nets $(\mathrm{AOR}=5.0 ; 95 \% \mathrm{CI}: 3.34-4.43)$ were significantly associated with malaria infection at migration phases.

Conclusion: This study showed that the burden of malaria among seasonal migrant workers was high at transit and destination phases. Malaria prevalence was higher at the destination phase compared to the transit phase. A tailored malaria prevention intervention is needed including awareness creation, screening, treatment, repellent, and prophylaxis at both phases to reduce malaria infections.
\end{abstract}

Keywords: destination, malaria, malaria prevalence, seasonal migrant farm workers, transit

\section{Introduction}

Despite years of global and country-level interventions, malaria continues to be a global public health problem. It is caused by Plasmodium parasites and in most cases transmitted through the bites of female Anopheles mosquitoes. ${ }^{1}$ Among the five Plasmodium parasite species which cause malaria in humans, Plasmodium falciparum and Plasmodium vivax are widely distributed in the tropical and subtropical countries of the world. In Africa, P. falciparum is the most prevalent cause
Correspondence: Tesfaye Tilaye

Tel +251 911677055

Email tilayebiru2020@gmail.com 
of malaria infection. ${ }^{2,3}$ Globally, between 2000 and 2019, a successful decline of malaria incidence was documented from 80 cases per 1000 population at risk to 57 cases per the same and total malaria cases declined from 238 million in 2000 to 229 million in 2009. The malaria death incidence rate decreased from 25 per 100,000 population at risk in 2000 to 10 in 2019. Moreover, in the last two decades, the total number of deaths declined by $60 \%$; from 736,000 in 2000 to 209,000 in $2019 .{ }^{4}$ The African region showed a remarkable decline in malaria deaths, 680,000 deaths in 2000 to 384,000 deaths in 2019. However, the region still carries $94 \%$ of the global burden of malaria cases. ${ }^{4}$

Over $68 \%$ of the Ethiopian landmass is malarious and about 60 million of the population is at risk. ${ }^{5-7}$ In 2016, an estimated three million new malaria cases and five thousand deaths were reported, showing a $50 \%$ decline in malaria incidence and mortality compared to the previous years. $^{8,9}$ This achievement was associated with an improved coverage of long-lasting insecticidal nets (LLINs), indoor residual spraying (IRS), malaria diagnoses using a rapid diagnostic test (RDT) and prompt treatment using artemisinin-based combination therapies (ACT), and destruction of mosquito breeding sites using environmental management implemented from 2005 to 2015. ${ }^{9}$ However, malaria remains among the ten top leading causes of morbidity and mortality in the country. ${ }^{10,11}$ Moreover, the country has not yet established a robust surveillance and health management information system to monitor the incidence and mortality rates of malaria. ${ }^{10}$

Most migration is either cyclical or seasonal in countries including Ethiopia. ${ }^{12}$ In Asia and Africa, people move from country to country or within a country for economic reasons, mostly for agricultural activities. ${ }^{13}$ Most agricultural farms are found in high malaria transmission areas and movement from low malaria transmission areas to these areas puts migrants at risk of infection. ${ }^{14}$ This results in resurgence of malaria, incidence of outbreaks, and spread of malaria parasites, including drug-resistant strains, to low malaria transmission areas through migrant workers by carrying these parasites with them while they return to their origin. This poses major challenges for malaria prevention and elimination activities. ${ }^{15,16}$ Other studies have shown that the risk of confirmed malaria in high land areas was up to seven times higher among people who traveled to high malaria transmission areas than those who did not. ${ }^{17,18}$ Also, gender (with male sex being more affected), ${ }^{19,20}$ low educational status, lack of knowledge on malaria prevention methods, ${ }^{16,21,22}$ sleeping outside, night-time work, low treatment seeking behavior, low utilization of insecticide-treated nets (ITNs), ${ }^{23,24}$ and low access to and utilization of ITNs ${ }^{14,20,25}$ were identified in studies as risk factors for malaria infection.

In Ethiopia, migration to mechanized farm areas is seasonal. ${ }^{25}$ Every year, an estimated 500,000 seasonal migrant workers move mostly from relatively low malaria endemic areas to development corridors during planting, weeding, and harvesting seasons (June-December). ${ }^{26}$ These seasons are characterized by high malaria transmission placing seasonal farm workers at significant risk of malaria infections during their travel, transit, and at destinations. Health facility-based malaria studies revealed a high prevalence of malaria parasites among travelers to malaria endemic areas. ${ }^{7,20}$ Moreover, study surveys conducted among seasonal migrants at destination sites indicated high malaria prevalence, almost nine-fold higher than the national malaria indicator survey result in $2015 .{ }^{14}$

Despite the high malaria burden and negative consequences of seasonal migration, less attention has been given to migrant workers in Ethiopia. Even though Ethiopia plans to eliminate malaria by the year 2030 targeting 239 districts with low malaria prevalence, the malaria prevention and control strategy of the country mainly focuses on stable and non-migrant populations. ${ }^{14}$ It is very important to understand the malaria burden among seasonal migrant workers to help consider migrants in the country's malaria elimination strategy.

The few studies conducted in Ethiopia focused on farm areas. However, the burden of malaria at origin, transit, and destination sites and the risks associated with malaria infection were not well addressed. This study aims to determine the prevalence of malaria infection and the risk factors associated with it at development corridors: transit and destination phases among seasonal migrant workers, in Northwest Ethiopia.

\section{Materials and Methods}

\section{Study Settings}

Development corridors are mostly found in lowland areas and are known for their high agricultural productivity. Every year, an estimated 500,000 seasonal migrant workers move mostly from relatively low malaria endemic areas to development corridors during planting, weeding, and harvesting seasons (June-December). ${ }^{26}$ These seasons 
are characterized by high malaria transmission, placing seasonal farm workers at significant risk of malaria infection during their travel, transit, and destinations. In Amhara Regional State of Ethiopia, there are nine development corridors in nine districts of Metema, West Armachiho, and Quara Districts in West Gondar Zone, Basso Liben, Debre Elias, and Gozamen Districts in East Gojam Zone, Jawi and Ayehu Guagusa in Awi Zone, and Tegede District in Central Gondar Zone. In these development corridors, hundreds of investors are involved in plantation of various agricultural products mostly sesame, sorghum, and cotton.

This study was conducted in Metema District in Northwest Ethiopia (Figure 1). It is one of the nine agricultural investment districts with a total permanent resident population of $154,618 .^{27}$ The district shares boundaries with Quara, West Armachiho, and Chilga Districts within the country as well as with Sudan. The study area is a lowland with an average altitude of 750 meters above sea level (range: 500-1000). The mean

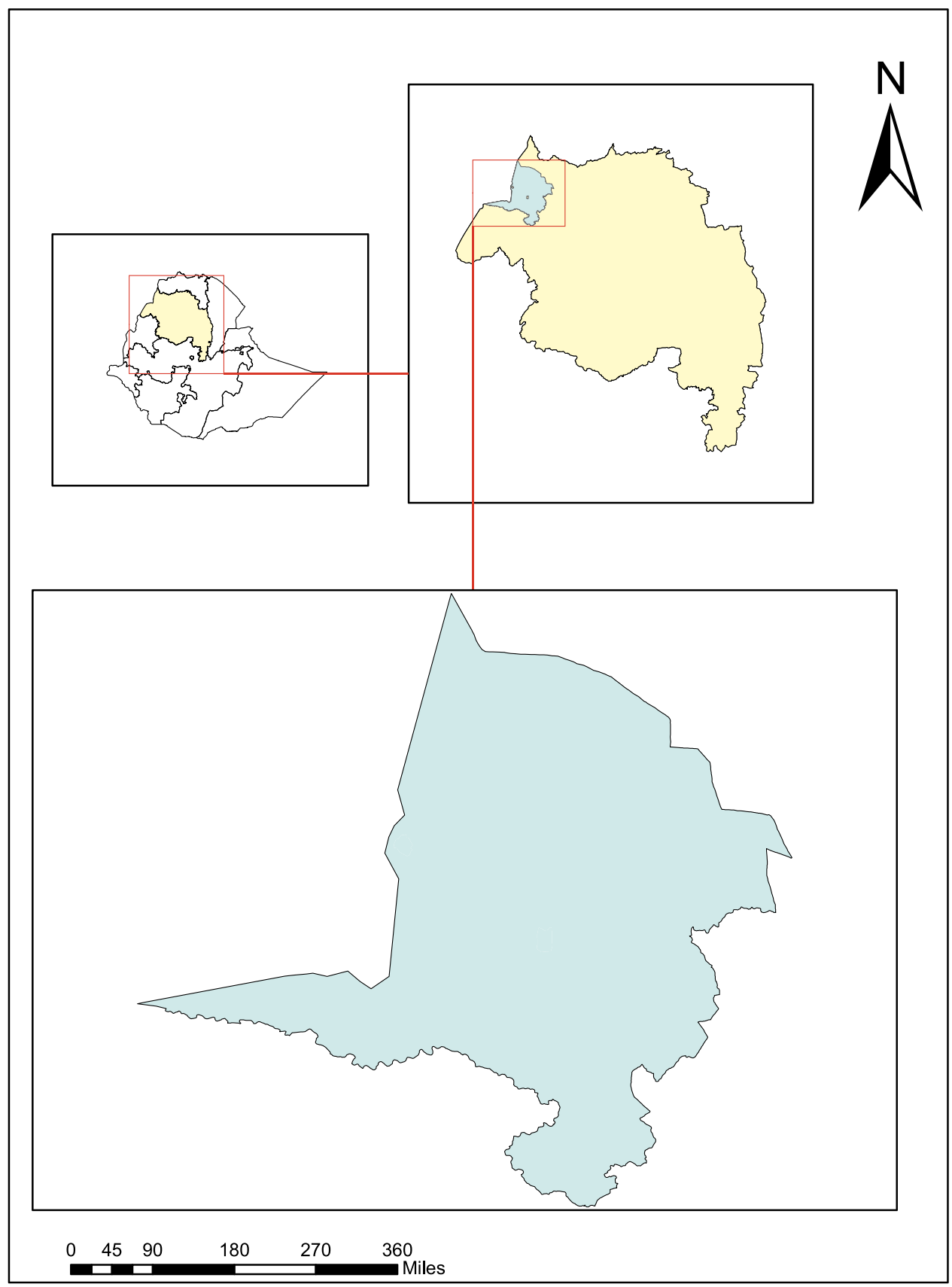

Figure I Map of Metema district, Amhara Region, Northwest Ethiopia, Ethiopia. 
annual rainfall ranges from about $850 \mathrm{~mm}$ to around $1000 \mathrm{~mm}$. The district has 26 rural and three urban kebeles - the lowest-level political administrative unit in Ethiopia. It also has one district public hospital, five health centers, and 26 health posts as well as private health facilities including 47 clinics, five medium diagnostic laboratories, 14 drug venders, nine rural drug shops, and 21 legal traditional medical centers. ${ }^{26}$

Metema district is one of the seven agricultural investment areas receiving an estimated 120,000 seasonal migrant workers mainly from Amhara Region with various climatic zones: highland, midland, and lowland. These migrants are mostly engaged in site clearing, land preparation, planting, and harvesting of sesame, sorghum, and cotton products at their destination.

Site clearing, land preparation, planting, and weeding take place from May to August. Harvesting of sesame is from the end of September to December when major malaria transmission occurs following the main rainy season (June-September). Some migrants remain at their destination from one to six months to harvest sorghum and cotton.

\section{Study Design and Period}

A repeated cross-sectional study was conducted at two migration phases to determine the prevalence of malaria and related risk factors among seasonal migrant farm workers. The first cross-sectional study was conducted at the transit phase from September 25 to October 5, 2018. All study subjects tested positive for malaria at transit were treated with antimalarial drugs according to the national guideline on malaria treatment. The second crosssectional study has been conducted at destination phase from October 30 to November 10, 2018. The time interval was set considering the two most common incubation periods of $P$. falciparum and $P$. vivax.

Transit in this study is where the migrants stay for some days until they find a job. Destination is the location where seasonal migrant workers stay for weeks or even months for planting and harvesting of sesame and other seasonal crops in the study area. For this study, destinations were Abederge, Ambase, Delelo Kuter 1, Delelo Kuter 2, Deteye Gebiru, Durba, Senar, and Tesfaye Worku farm areas.

\section{Sample Size Determination}

A single population proportion formula was used to determine the independent sample size for each migration phase. The following assumptions were taken to determine the sample sizes: $95 \%$ confidence interval, $2 \%$ margin of error, $10 \%$ of non-response rate, and $12 \%$ of malaria prevalence. ${ }^{28}$ Accordingly, the total calculated sample size was 1115 for each migration phase.

\section{Sampling Technique and Data Collection}

Five transit sites, Delelo town, Delelo Kuter 1, Metema Yohannes, Senar, and Kokit, were identified based on the information from Metema investment office and health office during the study period. These sites were common transit areas for migrant workers on their way to farm areas. More than $89 \%$ of migrants were using Delelo town, Delelo Kuter 1, and Metema Yohannes as a transit to reach different farm sites. The sample size was proportionally distributed to the transit sites based on the number of expected seasonal migrant workers in each transit. A simple random sampling technique was used to select study participants for interview and blood sample collection.

Abederge, Ambase, Delelo Kuter 1, Delelo Kuter 2, Deteye Gebiru, Durba, Senar, and Tesfaye Worku were identified as destination sites. A venue-based sampling technique was used to reach the subjects at these sites for interview. Time-space sampling was used for sampling hard to reach populations. ${ }^{29}$ Seasonal migrant workers were among the hard to reach populations with traditional sampling methods. This technique is an alternative to traditional probability sampling. ${ }^{30,31}$ Information on locations, times, and where and when to contact seasonal migrant farm workers were gathered from officials working at the Bureau of Environmental Protection, Land Administration and Use, Bureau of Labor and Social Affairs, and health officials at regional, zonal, and district levels. Site visits were conducted where discussions were held with farm owners or managers and workers. Then, seven farm areas and one town were identified to conduct the study. These areas were venues where nearly $96 \%$ of the seasonal migrant workers were found. In the seven farms, there were 66 blocks with the capacity of up to 30 farm sites with $300-500$ workers. A total of 40 blocks were selected using simple random sampling and the sample size was distributed proportionally to yield the desired number of study subjects based on the calculated sample size.

Study teams approached the first farm they encountered after entering the block and $40 \%$ of the gathered farmers were selected using systematic sampling for interview and blood sample collection. Farm sites with no 
seasonal migrants during the survey were replaced by adjacent farms within the selected block.

A structured questionnaire was used to collect data from 13 survey sites (five transit sites and eight destination sites) from suburban or farm sites for malaria infection. Sociodemographic characteristics, place of permanent residence, climatic zones (highland, midland, lowland), knowledge of malaria, knowledge of malaria prevention methods, and practice of malaria prevention were asked from each respondent.

For the data collection, ten data collectors were involved in the data collection from the sites. All data collectors have three to five-year experience of malaria data collection in the area. The quality of data collection was monitored daily by three supervisors and the principal investigator.

\section{Laboratory Diagnosis of Malaria Parasite}

Two drops of capillary blood samples were collected from each study participant following standard procedures. Both thick and thin blood smears were prepared on a clean microscopic slide from each selected seasonal migrant worker following standard operating procedures. ${ }^{32}$ One drop was used to prepare a thick smear and the other was used to prepare a thin smear. ${ }^{33}$ Finally, the slides were labeled with the participant's code and packed into slide porter after being air dried. ${ }^{34}$ All slides were transported to Metema Hospital located in Gendwuha town. The thin smear on each slide was fixed with absolute methanol and both thick and thin smears were stained with $10 \%$ Giemsa for 10 minutes, air dried, and examined under a light microscope for malaria parasites. Results were reported based on screening of 100 microscopic fields at $100 \times$ magnification. The initial thick film was classified as negative if no parasites was found after 500 white blood cell count. For quality assurance, $10 \%$ of positive slides were checked for species confirmation $^{35}$ by a senior laboratory technician who is a certified microscopist with 15 years of experience of malaria diagnosis at cluster health centers and at a district hospital of Metema district.

\section{Data Processing and Analysis}

Data were coded, double entered, and cleaned using EpiData version 3.1 software (EpiData Association, Odense, Denmark). The data were then exported to SPSS version 20 (IBM SPSS statistics for Windows; IBM Corp., Armonk, NY, USA) for analysis. Data quality was checked for completeness and consistency by running frequency and descriptive statistics.

After the quality check, descriptive statistics were calculated. A chi-square test was conducted to explore associations among categorical variables. Levels of association between various variables were determined by the Fisher's exact test in situations where the expected frequencies were less than five. Multivariate logistic regression was performed to test associations and relationships. Adjusted odds ratios were calculated. A $p$-value $<0.05$ was considered statistically significant.

\section{Results}

\section{Socio-Demographic Characteristics of Seasonal Migrant Workers}

A total of 2201 seasonal migrant workers were interviewed with a response rate of $100 \%$ (1115 respondents) at transit and $97.4 \%$ (1086) at destination phases, respectively.

Almost all, 2163 (98.3\%), of the respondents were male and the sex distribution was similar across the migration phases. The age of the respondents ranged from 15 to 71 years with a mean age of $26.5(7.3 \pm \mathrm{SD})$ years. The age distribution was similar for the 25-29 age category but varied for the rest of the age categories at transit and destination phases. The majority $(52.0 \%)$ of the respondents had no education and there was no difference in educational status between transit and destination phases. Of the total respondents at migration phases, 1107 (50.3\%) were daily laborers, 805 (36.6\%) were farmers, and 289 (13.1\%) were students. The majority, 1501 (68.2\%), were single with a higher proportion for destination, 785 (72.3\%), compared to transit, $716(64.2 \%)$, phases. The majority, $2163(98.3 \%)$, of the respondents were Orthodox Christians (Table 1).

\section{Prevalence of Malaria}

The prevalence of malaria infection was $13.5 \%$ (95\% CI: $12.07-14.93 \%$ ) and $18.7 \%$ (95\% CI: $16.40-21.02 \%)$ at transit and destination phases, respectively. The combined prevalence using microscopy was $16.1 \%$ (95\% CI: $14.67-$ 17.63\%). Of all the positive cases, $72.60 \%$ (95\% CI: $70.2-$ $75.0 \%$ ) of them were $P$. falciparum and $27.40 \%$ (95\% CI: 23.4-31.4\%) were P. vivax. Percentages of P. falciparum at transit and destination were $36.6 \%$ (95\% CI: $32.6-40.6 \%)$ and $63.4 \%(95 \% \mathrm{CI}: 60.4-66.4 \%)$, respectively and P. vivax was $58.8 \%$ (95\% CI: $55.5-62.1 \%$ ) and $41.2 \%$ 
Table I Socio-Demographic Characteristics of Seasonal Migrant Workers by Migration Phases, Metema District, Northwest Ethiopia, September 25-November 10, $2018(n=2201)$

\begin{tabular}{|c|c|c|c|}
\hline \multirow[t]{2}{*}{ Characteristics } & \multicolumn{2}{|c|}{ Migration Phases } & \multirow[b]{2}{*}{ Total (\%) } \\
\hline & Transit (\%) & Destination (\%) & \\
\hline \multicolumn{4}{|l|}{ Sex } \\
\hline Male & II0I (98.7) & $1062(97.8)$ & $2163(98.3)$ \\
\hline Female & $14(1.3)$ & $24(2.2)$ & $38(1.7)$ \\
\hline \multicolumn{4}{|l|}{ Age (years) } \\
\hline $15-19$ & $162(7.4)$ & $93(4.2)$ & $255(1 \mathrm{I} .6)$ \\
\hline $20-24$ & $346(15.7)$ & $404(18.4)$ & $750(34.1)$ \\
\hline $25-29$ & $285(\mid 2.9)$ & $282(12.8)$ & $567(25.8)$ \\
\hline $30-34$ & $128(5.8)$ & I89 (8.6) & $317(14.4)$ \\
\hline $35+$ & $194(8.8)$ & $118(5.4)$ & $312(14.2)$ \\
\hline \multicolumn{4}{|l|}{ Education } \\
\hline Formal education & $352(31.6)$ & $322(29.7)$ & $674(30.6)$ \\
\hline No education & $763(60.1)$ & $764(70.3)$ & 1527 (69.4) \\
\hline \multicolumn{4}{|l|}{ Occupation } \\
\hline Farmer & $671(60.2)$ & $436(40.1)$ & $805(36.6)$ \\
\hline Daily laborer & $298(26.7)$ & $507(46.7)$ & $1107(50.3)$ \\
\hline Student & $146(13.1)$ & $143(13.2)$ & $289(13.1)$ \\
\hline \multicolumn{4}{|l|}{ Marital status } \\
\hline Married & $359(32.2)$ & $272(25.0)$ & $631(28.7)$ \\
\hline Single & $716(64.2)$ & $785(72.3)$ & $|50|(68.2)$ \\
\hline Divorced/widow & $40(3.6)$ & $29(2.7)$ & $69(3.1)$ \\
\hline \multicolumn{4}{|l|}{ Religion } \\
\hline Orthodox & $1090(97.8)$ & $1073(98.8)$ & $2163(98.3)$ \\
\hline Others $^{\mathrm{a}}$ & $25(2.2)$ & $13(1.2)$ & $38(1.7)$ \\
\hline \multicolumn{4}{|l|}{ Ethnicity } \\
\hline Amhara & II 02 (98.8) & $1075(99.0)$ & 2177 (98.9) \\
\hline Others $^{b}$ & $13(1.1)$ & II (I.0) & $24(I . I)$ \\
\hline
\end{tabular}

Notes: a'Muslim, Protestant; 'Agew, Oromo, South, Tigray.

(95\% CI: 37.3-45.1\%) for transit and destination, respectively. Presence of malaria infection and infective Plasmodium parasite were significantly associated with migration phases $\left(X^{2}=23.316, P=0.0001\right)$ (Table 2).

\section{Knowledge of Malaria Prevention and Control}

Knowledge of malaria prevention and control methods were assessed at transit and destination phases. Accordingly, the majority of the respondents (73.6\%) mentioned one to three malaria drugs currently in use for malaria treatment according to Ethiopian malaria treatment guidelines. Knowledge of antimalarial drugs was significantly higher among respondents at the destination phase
(76.5\%) compared to respondents at the transit phase (70.9\%) $(P=0.003)$. The overall knowledge of IRS was $64.3 \%$ and it was similar for transit $(65.4 \%)$ and destination (63.3\%) phases. Knowledge of LLINs at transit was significantly higher than the destination phase $\left(X^{2}=21.945\right.$, $P=0.0001)$. The overall knowledge of draining stagnant water $(28.0 \%)$, clearing vegetation $(24.5 \%)$, closing openings $(8.1 \%)$, repellents $(10.1 \%)$, and smoking $(11.6 \%)$ was low at both migration phases. Knowledge of draining stagnant water $\left(X^{2}=10.461, P=0.001\right)$, clearing vegetation $\left(X^{2}=7.62, \quad P=0.006\right), \quad$ closing openings $\quad\left(X^{2}=11.434\right.$, $P=0.001)$, and repellents $\left(X^{2}=68.503, P=0.001\right)$ was higher at destination and the difference was statistically significant (Table 3).

\section{Malaria Prevention Practices Among Seasonal Migrant Workers at Migration Phases}

The overall LLIN ownership at migration phases was 566 (25.7\%). Ownership of LLINs was 389 (34.6\%) and 177 $(16.3 \%)$ at transit and destination phases, respectively. The majority, 1635 (74.3\%), of the seasonal migrant workers had no LLINs at both migration phases. Among those who owned LLINs, 546 (96.5\%) of the respondents were using LLINs during the study period. In this case, the majority, 379 (97.4\%), of the study subjects at transit were using LLINs. The frequency of LLIN utilization was similar for transit and destination, 301 (79.4\%) and $132(79.0 \%)$, phases, respectively. About 35 (21\%) of the respondents occasionally utilize LLINs. The use of LLINs in the last one month at transit and destination phases was 303 (79.8\%) and 157 (94.0\%), respectively. This study indicated that very few seasonal migrants were using repellents, 58 (2.6\%), wearing long sleeve clothes, 452 (20.5\%), and smoking, 160 (7.3\%), to prevent malaria infection due to Anopheles mosquito bites. Ownership of LLINs $\left(X^{2}=99.52, P=0.0001\right)$, and LLIN use in the last one month $\left(X^{2}=17.28, P=0.0001\right)$ were associated with migration phases (Table 4).

\section{Multivariable Analysis Using Logistic}

\section{Regression Model}

Education, knowledge of antimalarial drugs, IRS, and LLINs were significant predictors of malaria infection at the migration phases. Noneducated seasonal migrant farm workers at destination had eight times higher risk for malaria infection compared to seasonal migrant farm 
Table 2 Prevalence of Malaria and Infecting Plasmodium Species by Migration Phases Among Seasonal Migrant Workers at Migration Phases, Metema District, Northwest Ethiopia, September 25-November 10, $2018(n=2201)$

\begin{tabular}{|l|c|c|c|c|c|}
\hline \multirow{2}{*}{ Species } & \multicolumn{3}{|c|}{ Migration Phases } & \multirow{2}{*}{$X^{2}$} & \multirow{2}{*}{ P value } \\
\cline { 2 - 5 } & Transit n (\%) & Destination n (\%) & $\begin{array}{c}\text { Transit + Destination } \\
\text { n (\%) }\end{array}$ & \\
\cline { 2 - 4 } & Microscopy & Microscopy & Microscopy Total (\%) & \multirow{2}{*}{23.316} & \\
\hline Total negative & $964(86.5 \%)$ & $883(81.3 \%)$ & $1847(83.9 \%)$ & & \\
\hline Total positive & $151(13.5 \%)$ & $203(18.7 \%)$ & $354(16.1 \%)$ & \\
\hline P. falciparum & $94(26.6 \%)$ & $163(46.0 \%)$ & $257(72.6 \%)$ & \\
\hline P. vivax & $57(16.1 \%)$ & $40(11.3 \%)$ & $97(27.4 \%)$ & \\
\hline
\end{tabular}

Table 3 Knowledge of Malaria Prevention and Control Among Seasonal Migrant Workers at Migration Phases, Metema District, West North Ethiopia, September 25-November 10, 2018 ( $n=2201$ )

\begin{tabular}{|c|c|c|c|c|c|}
\hline \multirow[t]{2}{*}{ Characteristics } & \multicolumn{2}{|c|}{ Migration Phases } & \multirow[t]{2}{*}{ Total n (\%) } & \multirow[t]{2}{*}{$x^{2}$} & \multirow[t]{2}{*}{$P$ value } \\
\hline & Transit n (\%) & Destination n (\%) & & & \\
\hline \multicolumn{6}{|l|}{ Antimalarial drugs } \\
\hline Yes & 790 (70.9) & 831 (76.5) & 1621 (73.6) & 9.105 & 0.003 \\
\hline No & $325(29.1)$ & $255(23.5)$ & $580(26.4)$ & & \\
\hline \multicolumn{6}{|l|}{ IRS } \\
\hline Yes & $729(65.4)$ & $687(63.3)$ & $1416(64.3)$ & 1.097 & 0.299 \\
\hline No & $386(34.6)$ & $399(36.7)$ & 785 (35.7) & & \\
\hline \multicolumn{6}{|l|}{ LLINs } \\
\hline Yes & $706(63.3)$ & $58 \mid(53.5)$ & $1287(58.5)$ & 21.845 & 0.0001 \\
\hline No & 409 (36.7) & $505(46.5)$ & $9 \mid 4(4 \mid .5)$ & & \\
\hline \multicolumn{6}{|l|}{ Drain stagnant water } \\
\hline Yes & $278(24.9)$ & $338(31.1)$ & $616(28.0)$ & $|0.46|$ & 0.001 \\
\hline No & $837(75.1)$ & $748(68.9)$ & $1585(72.0)$ & & \\
\hline \multicolumn{6}{|l|}{ Clearing vegetation } \\
\hline Yes & 255 (22.9) & $304(28.0)$ & $559(25.4)$ & 7.62 & 0.006 \\
\hline No & $860(77.1)$ & $782(72.0)$ & $1642(74.6)$ & & \\
\hline \multicolumn{6}{|l|}{ Closing openings } \\
\hline Yes & $69(6.2)$ & $110(10.1)$ & $179(8.1)$ & 11.434 & 0.001 \\
\hline No & $1046(93.8)$ & $976(89.9)$ & 2022 (91.9) & & \\
\hline \multicolumn{6}{|l|}{ Repellent } \\
\hline Yes & $54(4.8)$ & $168(15.5)$ & $222(10.1)$ & 68.503 & 0.0001 \\
\hline No & I06I (95.2) & $918(84.5)$ & $1979(89.9)$ & & \\
\hline \multicolumn{6}{|l|}{ Smoking } \\
\hline Yes & $123(11.0)$ & $133(12.2)$ & $256(11.6)$ & 0.791 & 0.374 \\
\hline No & $992(89.0)$ & $953(87.8)$ & $1945(88.4)$ & & \\
\hline
\end{tabular}

Abbreviations: IRS, indoor residual spraying; LLINs, long-lasting insecticidal nets.

workers who had formal education $(\mathrm{AOR}=8.198$ (95\% CI: 4.318-15.564). Seasonal migrant workers who had no knowledge of antimalarial drugs at the destination phase were 2.4 times at higher risk for malaria infection compared to seasonal migrant farm workers with good knowledge of antimalaria drugs ( $\mathrm{AOR}=2.4 ; 95 \% \mathrm{CI}: 1.43-3.95)$. 
Table 4 Malaria Prevention Practice at Migration Phases, Metema District, Northwest Ethiopia, September 25- November 10 , 20I8 $(n=2201)$

\begin{tabular}{|c|c|c|c|c|c|}
\hline \multirow[t]{2}{*}{ Characteristics } & \multicolumn{2}{|c|}{ Migration Phases } & \multirow[t]{2}{*}{ Total n (\%) } & \multirow[b]{2}{*}{$x^{2}$} & \multirow[b]{2}{*}{$P$ value } \\
\hline & Transit n (\%) & Destination n (\%) & & & \\
\hline $\begin{array}{l}\text { LLIN ownership } \\
\text { Yes } \\
\text { No }\end{array}$ & $\begin{array}{l}389(34.6) \\
726(65.1)\end{array}$ & $\begin{array}{l}177(16.3) \\
909(83.7)\end{array}$ & $\begin{array}{c}566(25.7) \\
1635(74.3)\end{array}$ & 99.52 & 0.0001 \\
\hline $\begin{array}{l}\text { Using LLINs } \\
\text { Yes } \\
\text { No }\end{array}$ & $\begin{array}{c}379(97.4) \\
10(2.6)\end{array}$ & $\begin{array}{c}167(94.4) \\
10(5.6)\end{array}$ & $\begin{array}{c}546(96.5) \\
20(3.5)\end{array}$ & 3.38 & 0.066 \\
\hline $\begin{array}{l}\text { LLIN using frequency } \\
\text { Daily } \\
\text { Sometimes }\end{array}$ & $\begin{array}{c}30 \mathrm{I}(79.4) \\
78(20.6)\end{array}$ & $\begin{array}{c}\mid 32(79.0) \\
35(21.0)\end{array}$ & $\begin{array}{l}433(79.3) \\
\text { I I } 3(20.7)\end{array}$ & 0.01 & 0.92 \\
\hline $\begin{array}{l}\text { LLINs used in the last month } \\
\text { Yes } \\
\text { No }\end{array}$ & $\begin{array}{c}303(79.9) \\
76(20.1)\end{array}$ & $\begin{array}{c}157(94.0) \\
10(6.0)\end{array}$ & $\begin{array}{c}460(84.2) \\
86(15.8)\end{array}$ & 17.28 & 0.0001 \\
\hline $\begin{array}{l}\text { Using repellent } \\
\text { Yes } \\
\text { No }\end{array}$ & $\begin{array}{c}27(2.4) \\
1088(97.6)\end{array}$ & $\begin{array}{c}31(2.9) \\
1055(97.1)\end{array}$ & $\begin{array}{c}58(2.6) \\
2143(97.4)\end{array}$ & 0.40 & 0.526 \\
\hline $\begin{array}{l}\text { Wearing long sleeves } \\
\text { Yes } \\
\text { No }\end{array}$ & $\begin{array}{l}275(24.7) \\
840(75.3)\end{array}$ & $\begin{array}{l}177(16.3) \\
909(83.7)\end{array}$ & $\begin{array}{l}452(20.5) \\
1749(79.5)\end{array}$ & 23.59 & 0.0001 \\
\hline $\begin{array}{l}\text { Smoking } \\
\text { Yes } \\
\text { No }\end{array}$ & $\begin{array}{c}91(8.2) \\
1024(91.8)\end{array}$ & $\begin{array}{c}69(6.4) \\
1017(93.6)\end{array}$ & $\begin{array}{c}160(7.3) \\
204 \mid(92.7)\end{array}$ & 2.667 & 0.102 \\
\hline
\end{tabular}

Abbreviation: $n$, number.

Moreover, migrants who had no knowledge of LLINs at the destination had five times higher risk of malaria infection (AOR=5.0; 95\% CI: 3.34-4.43). However, knowledge of IRS at transit was found to be protective for seasonal migrant workers from getting malaria infection $(\mathrm{AOR}=0.7$; 95\% CI: 0.46-0.98) (Table 5).

\section{Discussion}

This study determined malaria prevalence and related risk factors at development corridors in Northwest Ethiopia. Educational status, knowledge of antimalarial drugs, and use or non-use of long-lasting insecticidal nets and insecticide residual spray were significantly associated with malaria infection.

In this study, the burden of malaria infection was generally high in both transit and destination migration phases while malaria prevalence varied between phases. Overall, a high prevalence of malaria was detected among seasonal migrant farm workers. Malaria prevalence in this study was higher than the malaria prevalence determined among seasonal migrant workers during the plantation and weeding season in Ethiopia, ${ }^{14} \mathrm{DRC}^{36}$ and Thailand. ${ }^{37}$ The current study was conducted from September to November 2018 during a major malaria transmission season, which might have contributed to the high malaria prevalence compared to the study conducted during the rainy season in the same geographic area using a similar study tool. Moreover, the malaria prevalence finding of this study was lower than malaria prevalence study findings conducted in Ethiopia, ${ }^{38-40}$ Ghana, ${ }^{41}$ and DRC. $^{42,43}$ The difference might be associated with the study subjects, measurement tools, and study areas.

The study revealed high combined prevalence of P. falciparum and a relatively high proportion of $P$. vivax at transit sites. This finding is comparable with a prior study conducted in Northwest Ethiopia, ${ }^{38}$ higher than the study findings in Southwest Ethiopia, ${ }^{14,44,45}$ Ghana, ${ }^{41}$ India, ${ }^{46}$ and Thailand $^{37}$ but lower than the prevalence 
Table 5 Predictors of Plasmodium Infection Risk Among Seasonal Migrant Farm Workers at Migration Phases in Metema District, West North Ethiopia, September 25-November 10, 2018 ( $n=2201)$

\begin{tabular}{|c|c|c|c|c|c|c|c|c|}
\hline \multirow[t]{2}{*}{ Characteristics } & \multicolumn{4}{|c|}{ Transit } & \multicolumn{4}{|c|}{ Destination } \\
\hline & $\mathbf{N}$ & AOR & $95 \% \mathrm{Cl}$ & $P$ value & $\mathbf{N}$ & AOR & $95 \% \mathrm{Cl}$ & $P$ value \\
\hline \multicolumn{9}{|l|}{ Age (years) } \\
\hline $15-19$ & 162 & 1 & & & 93 & 1 & & \\
\hline $20-24$ & 346 & $\mathrm{I} .4 \mathrm{I}$ & $0.78-2.54$ & 0.25 & 404 & 1.17 & $0.56-2.44$ & 0.68 \\
\hline $25-29$ & 285 & 1.21 & $0.65-2.23$ & 0.85 & 282 & 1.07 & $0.64-1.79$ & 0.79 \\
\hline $30-34$ & 128 & 1.13 & $0.54-2.34$ & 0.75 & 189 & 0.75 & $0.43-1.29$ & 0.29 \\
\hline $35+$ & 194 & 1.59 & $0.85-3.01$ & 0.15 & 118 & 1.03 & $0.59-1.79$ & 0.92 \\
\hline \multicolumn{9}{|c|}{ Educational status } \\
\hline Formal education & 352 & 1 & & & 322 & 1 & & \\
\hline No education & 763 & 1.46 & $0.97-2.19$ & 0.07 & 764 & 8.19 & $4.32-15.56$ & 0.0001 \\
\hline \multicolumn{9}{|c|}{ Occupational status } \\
\hline Farmer & 671 & 1 & & & 436 & 1 & & \\
\hline Daily laborer & 298 & 0.9 & $0.59-1.37$ & 0.63 & 507 & 1.14 & $0.79-1.66$ & 0.5 \\
\hline Student & 146 & 0.84 & $0.43-1.64$ & 0.62 & 143 & 0.96 & $0.48-1.93$ & 0.91 \\
\hline \multicolumn{9}{|l|}{ Marital status } \\
\hline Married & 359 & 1 & & & 272 & 1 & & \\
\hline Single & 716 & 1.02 & $0.66-1.67$ & 0.92 & 785 & 1.06 & $0.69-1.64$ & 0.78 \\
\hline Divorced/widow & 40 & 0.85 & $0.3 \mathrm{I}-2.29$ & 0.74 & 29 & 1.43 & $0.54-3.78$ & 0.47 \\
\hline \multicolumn{9}{|c|}{ Knowledge of malaria prevention methods } \\
\hline \multicolumn{9}{|c|}{ Antimalarial drugs } \\
\hline Yes & 790 & 1 & & & 831 & 1 & & \\
\hline No & 325 & 1.18 & $0.76-1.83$ & 0.47 & 255 & 2.38 & $1.43-3.95$ & 0.001 \\
\hline \multicolumn{9}{|l|}{ IRS } \\
\hline Yes & 729 & 1 & & & 687 & 1 & & \\
\hline No & 386 & 0.67 & $0.46-0.98$ & 0.04 & 399 & 0.93 & $0.63-1.37$ & 0.69 \\
\hline \multicolumn{9}{|l|}{ LLINs } \\
\hline Yes & 706 & 1 & & & 581 & I & & \\
\hline No & 409 & 1.33 & $0.89-2.00$ & 0.16 & 505 & 5.03 & $3.34-7.56$ & 0.0001 \\
\hline \multicolumn{9}{|c|}{ Drain stagnant water } \\
\hline Yes & 278 & 1 & & & 338 & 1 & & \\
\hline No & 837 & 0.88 & $0.58-1.32$ & 0.55 & 748 & 0.82 & $0.56-1.20$ & 0.31 \\
\hline \multicolumn{9}{|c|}{ Clearing vegetation } \\
\hline Yes & 255 & 1 & & & 304 & 1 & & \\
\hline No & 860 & 0.99 & $0.63-1.55$ & 0.97 & 782 & 0.98 & $0.66-1.48$ & 0.97 \\
\hline \multicolumn{9}{|l|}{ Repellent } \\
\hline Yes & 54 & 1 & & & 168 & I & & \\
\hline No & 1061 & 1.29 & $0.37-4.44$ & 0.69 & 918 & 0.95 & $0.33-2.77$ & 0.93 \\
\hline \multicolumn{9}{|c|}{ Practice of malaria prevention methods } \\
\hline \multicolumn{9}{|l|}{ LLIN use } \\
\hline Daily & 301 & 1 & & & 132 & 1 & & \\
\hline Some times & 88 & 6.26 & $3.00-13.03$ & 0.001 & 45 & 1.79 & $0.18-17.96$ & 0.62 \\
\hline
\end{tabular}


Table 5 (Continued).

\begin{tabular}{|c|c|c|c|c|c|c|c|c|}
\hline \multirow[t]{2}{*}{ Characteristics } & \multicolumn{4}{|c|}{ Transit } & \multicolumn{4}{|c|}{ Destination } \\
\hline & $\mathbf{N}$ & AOR & $95 \% \mathrm{Cl}$ & $P$ value & $\mathbf{N}$ & AOR & $95 \% \mathrm{Cl}$ & $P$ value \\
\hline LLINs used in the last month & & & & & & & & \\
\hline Yes & 303 & I & & & 157 & I & & \\
\hline No & 86 & 0.93 & $0.43-2.00$ & 0.85 & 20 & 12.9 & $1.43-116.78$ & 0.23 \\
\hline Wearing long sleeves & & & & & & & & \\
\hline Yes & 275 & 1.1 & $0.73-1.65$ & 1.09 & 177 & 0.8 & $0.52-1.15$ & 0.21 \\
\hline No & 840 & I & & & 909 & I & & \\
\hline
\end{tabular}

reported in Sudan. ${ }^{47}$ Travel away from home for seasonal farm activities might have exposed seasonal migrant workers to high risk of $P$. falciparum infection. Harvest activities in the evening, ${ }^{48}$ no or little use of malaria prevention methods, ${ }^{49,50}$ and low malaria immunity could have exposed more seasonal migrant workers to malaria infection at destination than at transit. ${ }^{7,25,51}$

High prevalence of $P$. vivax at transit was in agreement with an institution-based study in Ethiopia. ${ }^{52}$ This might be due to the relapse of disease from previous infection which could have resulted following a single mosquito inoculation, by recrudescence and re-infection from a new mosquito inoculation. ${ }^{53}$ Moreover, in areas with high temperature, the relapse of $P$. vivax was more frequent and occurring with a shorter interval. ${ }^{54,55}$ Most study subjects who were studied at transit might be from areas with stable malaria transmission where $P$. vivax could be more prevalent. ${ }^{56}$ Furthermore, one of the studies conducted in Ethiopia revealed higher prevalence of $P$. vivax in highland areas. So, the chance of migrants from highland areas carrying the parasite with them to migration phases including transits was high. ${ }^{57}$ Thus, migrant workers from high malaria areas with a history of $P$. vivax infection could contribute to importation of $P$. vivax to the development corridors and spread the infection to the susceptible migrant workers.

Moreover, the country's strategy for malaria prevention and control, which does not include a focus on seasonal migrant workers, ${ }^{14}$ and exclusion of agricultural farm workers in a malaria elimination program ${ }^{58}$ might have increased their exposure to malaria infection. Seasonal migrant workers who traveled from midland and lowland malarious areas to study areas could also contribute to increase malaria prevalence both at transit and destination phases. ${ }^{7,14}$ High malaria prevalence at the destination was in agreement with a study conducted among asymptomatic migrants in West Armachiho District in Northwest Ethiopia. ${ }^{38}$ However, it was higher than malaria prevalence survey findings in Ethiopia, ${ }^{14}$ India, ${ }^{59}$ and Ghana ${ }^{60}$ and lower than the malaria prevalence finding reported from Cameroon. ${ }^{61}$

In this study, educational status of seasonal migrant workers was associated with malaria infection at the destination. The odds of malaria infection among noneducated migrant workers was 8.1 times higher compared to migrant workers with formal education. The finding was consistent with the study conducted in West Armachiho District of Northwest Ethiopia. ${ }^{38}$ This might be due to the fact that most of the respondents in the second wave of farm activities (harvesting) were daily laborers and farmers who had no formal education. This could affect their seeking for malaria service while they are sick or traveling to malaria endemic areas for agricultural activities. ${ }^{62}$ Better education holds the key to a sustainable response to malaria prevention and treatment services. ${ }^{62}$ Lack of education compromises access to information and knowledge of malaria prevention and control methods and the acceptance and the implementation of malaria safe practices. $^{62-64}$ Other, studies also showed the significant contribution of education to decreased malaria prevalence. $^{65-68}$ However, age, occupation, and marital status were not significantly associated with risk of malaria infection at both transit and destination phases.

In this study, lack of knowledge of antimalarial drugs was associated with risk of malaria infection at the destination phase. This might be due to lack of access to malaria information on the importance of antimalarial drugs at the destination phase. In this second wave of 
seasonal migration time, antimalarial drugs were found to be the common malaria prevention methods in the development corridors which could also increase the knowledge of seasonal farm workers. Moreover, migrants from malaria areas could have better information, exposure, and understanding of malaria prevention methods including antimalaria drugs. ${ }^{69-71}$ Poor knowledge of malaria prevention has been shown to be a risk factor for malaria infection and was in agreement with this finding. ${ }^{19}$ Knowledge of antimalarial drugs could also be affected by their migration status, educational level, and beliefs. ${ }^{72}$

Knowledge of IRS was associated with low risk of malaria infection at transit when compared to destination. This could be linked to information shared at transit on malaria prevention methods and IRS practices at transit sites. Rental houses in the towns used as transit to development corridors are usually sprayed by insecticide chemicals during the high malaria transmission season, which could also contribute to an awareness of IRS among seasonal migrant farm workers. Knowledge of malaria transmission among seasonal migrant farm workers can greatly contribute to prevention and success of control measures. $^{73,74}$

Lack of knowledge of LLINs was significantly associated with high risk of malaria infection among study subjects at the destination who were three times more likely to have malaria compared to study subjects who had knowledge of LLINs. The finding was in agreement with the study conducted elsewhere in Ethiopia. ${ }^{75,76}$ Proper knowledge of LLINs determines the perception of the effectiveness and usefulness of LLINs, effective use of LLINs, perceived benefits of using LLINs, and acceptability and compliance with net use. ${ }^{77,78}$ Inadequate knowledge of malaria prevention methods negatively influence practices of prevention methods. ${ }^{79}$ Although good knowledge of LLINs is not a guarantee for utilization of LLINs, in most cases people with good knowledge of LLINs have ensured better utilization of LLINs. Long-lasting insecticidal nets are a highly effective means of preventing malaria infection and reducing associated morbidity and mortality, particularly in endemic areas. ${ }^{80}$ Across subSaharan Africa, the use of LLINs has been shown to result in parasite prevalence reduction. ${ }^{69,81}$ However, individuals' knowledge and beliefs related to LLINs can influence LLIN use. $^{82}$

This study kept its strength by taking a large sample size, and using a repeated cross-sectional study design and sampling techniques to minimize selection bias and ensure internal and external validity. It was conducted at seasonal migrant workers' destination sites during harvest, the main malaria transmission season, based on the recommendation by the malaria survey conducted in 2015, which was carried out in plantation time.

Microscopy, the gold standard for malaria diagnosis, which is recommended for research purposes, was used to determine malaria prevalence by senior and licensed laboratory technicians. ${ }^{83}$

Our study limitations were: 1) the LLIN use data were collected based on self-reporting; observation was not done. This might have caused social desirability bias due to a high chance of choosing "yes" rather than "no" in search of approval or the reverse by expecting LLIN donation. To minimize the bias, the interview included the use of LLINs in the night before the date of interview and asked their permission to check whenever possible. 2) This study did not cover the malaria status of the seasonal migrant worker's origin, so this might increase the malaria prevalence result at different migration phases. However, the study tried to minimize the potential bias by interviewing the history of having malaria infection at the origin and excluded those who took prophylaxis two weeks prior to the study.

\section{Conclusion}

The prevalence of malaria is high among seasonal migrant workers at both transit and destination migration phases. There was significant difference in the magnitude of malaria between transit and destination phases. The chance of new malaria infection at destination is higher compared to transit. Lack of education, knowledge of antimalarial drugs, and knowledge of LLINs were associated with malaria infection in the study area. Malaria information, screening, and treatment are pivotal at migration phases, particularly at transit and departure sites to minimize the risk of malaria infection at the destination and the chance of carrying malaria to their home towns. The ownership of LLIN and use of LLINs are very low at the destination. So, alternative prevention methods like repellent should be promoted, especially during harvest time. Moreover, malaria prevention and control strategies for seasonal migrant workers shall consider the integration of origin, transit, destination, and departure. Finally, we recommend a cohort study to determine the incidence of malaria infection at migration phases. 


\section{Data Sharing Statement}

Data and all the necessary materials are available from the corresponding author upon request.

\section{Ethical Approval and Consent to Participate}

Ethical clearance was obtained from the institutional review board of University of Gondar on May 24, 2018 with the Ref. No. O/V/P/RCS/05/1301/2018. Written permission was obtained from Ethical Committee of Amhara Regional Health Bureau (ARHB), West Gondar Zonal Health Department, and Metema Woreda Health Office. Local administration and farm owners/administrators provided oral permissions. During data collection, informed consent was sought from all the study participants and they were informed and assured that interviews and blood tests were completely voluntary and written informed consent was obtained from study participants after the study objective was explained to them. The ethical review committee approved that study subjects under the legal age ( $<18$ years) could provide written informed consent on their own behalf for they moved independently to the farm areas and were not accompanied by their parents. The blood test was done free of charge. Malaria cases were treated freely according to the national malaria guidelines. All data and information from study participants were kept confidential. None of the study participant was listed by name. The study was conducted in accordance with the Declaration of Helsinki.

\section{Acknowledgment}

We are grateful to Ethiopian Public Health Institute for funding and Dr Ebba Abate, Dr Beyene Moges, $\mathrm{Mr}$ Adugna Dadi, and Mr Yohannes Dugasa for facilitating the fund. We also appreciate Dr Tariku Nigatu, Dr Abebaw Gebeyehu, and Dr Abebe Genetu for editorial support; and Metema district health office, particularly $\mathrm{Mr}$ Abebaw Yeshaneh for unreserved cooperation and facilitation of the data collection. We also thank data collectors and supervisors for their diligent work and study participants for their time and cooperation. Last not the least, my family and IPH staff for all support.

\section{Author Contributions}

All authors made a significant contribution to the work reported, whether that is in the conception, study design, execution, acquisition of data, analysis and interpretation, or in all these areas; took part in drafting, revising, or critically reviewing the article; gave final approval of the version to be published; have agreed on the journal to which the article has been submitted; and agree to be accountable for all aspects of the work.

\section{Funding}

This study was funded by Public Health Emergency Management Directorate, Ethiopian Public Health Institute, Federal Ministry of Health, Addis Ababa.

\section{Disclosure}

The authors report no conflicts of interest in this work.

\section{References}

1. World Health Organization. World Malaria Report 2014. Geneva, Switzerland: World Health Organization; 2014.

2. Dufera M, Dabsu R, Tiruneh G. Assessment of malaria as a public health problem in and around Arjo Didhessa sugar cane plantation area, Western Ethiopia. BMC Public Health. 2020;20:1-10. doi:10.1186/s12889-020-08784-5

3. World Health Organization. World Malaria Report 2019. Geneva, Switzerland: World Health Organization; 2019.

4. WHO. World Malaria Report 2020. 20 years of global progress and challenges. Geneva, Switzerland: WHO; 2020.

5. Nega D, Dana D, Tefera T, Eshetu T. Prevalence and predictors of asymptomatic malaria parasitemia among pregnant women in the rural surroundings of Arbaminch Town, South Ethiopia. PLoS One. 2015;10(4):e0123630. doi:10.1371/journal.pone.0123630

6. Haile M, Lemma H, Weldu Y. Population movement as a risk factor for malaria infection in high-altitude villages of Tahtay-Maychew District, Tigray, Northern Ethiopia: a Case-Control Study. Am J Trop Med Hyg. 2017;97(3):726-732. doi:10.4269/ajtmh.17-0129

7. Malede A, Alemu K, Aemero M, Robele S, Kloos H. Travel to farms in the lowlands and inadequate malaria information significantly predict malaria in villages around Lake Tana, Northwest Ethiopia: a matched case-control study. Malar J. 2018;17(1):290. doi:10.1186/ s12936-018-2434-y

8. Health. FDRoEMo. Ethiopian national malaria indictor survey 2011: technical summary Ethiopian: Ministry of Health of Ethiopia; 2012.

9. Yaya S, Bishwajit G, Ekholuenetale M, Shah V, Kadio B, Udenigwe O. Knowledge of prevention, cause, symptom and practices of malaria among women in Burkina Faso. PLoS One. 2017;12 (7):e0180508. doi:10.1371/journal.pone. 0180508

10. Deribew A, Dejene T, Kebede B, et al. Incidence, prevalence and mortality rates of malaria in Ethiopia from 1990 to 2015: analysis of the global burden of diseases 2015. Malar J. 2017;16(1):1-7. doi:10.1186/s12936-017-1919-4

11. Girum T, Shumbej T, Shewangizaw M. Burden of malaria in Ethiopia, 2000-2016: findings from the Global Health Estimates 2016. Trop Dis Travel Med Vaccines. 2019;5(1):11. doi:10.1186/ s40794-019-0090-z

12. IOM. A Global Report on Population Mobility and Malaria: moving towards elimination with migration in mind; 2013.

13. Lambin EF, Meyfroidt P. Global land use change, economic globalization, and the looming land scarcity. Proc Natl Acad Sci. 2011;108 (9):3465-3472. doi:10.1073/pnas.1100480108 
14. Schicker R, Hiruy N, Melak B, et al. A Venue-Based Survey of Malaria, Anemia and Mobility Patterns among Migrant Farm Workers in Amhara Region, Ethiopia. PLoS One. 2015;10(11): e0143829. doi:10.1371/journal.pone.0143829

15. Cotter C, Sturrock H, Mea H. The changing epidemiology of malaria elimination: new strategies for new challenges. Lancet Infect Dis. 2013;382:900-911.

16. Tatem AJ, Jia P, Ordanovich D, et al. The geography of imported malaria to non-endemic countries: a meta-analysis of nationally reported statistics. Lancet Infect Dis. 2017;17(1):98-107. doi:10.1016/S1473-3099(16)30326-7

17. Lynch CA, Bruce J, Bhasin A, Roper C, Cox J, Abeku TA. Association between recent internal travel and malaria in Ugandan highland and highland fringe areas. Trop Med Int Health. 2015;20 (6):773-780. doi:10.1111/tmi.12480

18. Tesfaye S, Belyhun Y, Teklu T, Mengesha T, Petros B. Malaria prevalence pattern observed in the highland fringe of Butajira, Southern Ethiopia: a longitudinal study from parasitological and entomological survey. Malar J. 2011;10(1):153. doi:10.1186/1475-2875-10-153

19. Chaveepojnkamjorn W, Pichainarong N. Behavioral factors and malaria infection among the migrant population, Chiang Rai province. J Med Assoc Thai. 2005;88(9):1293-1301.

20. Alemu K, Worku A, Berhane Y, Kumie A. Spatiotemporal clusters of malaria cases at village level, northwest Ethiopia. Malar J. 2014;13:223. doi:10.1186/1475-2875-13-223

21. Phyo Than W, Oo T, Wai KT, et al. Knowledge, access and utilization of bed-nets among stable and seasonal migrants in an artemisinin resistance containment area of Myanmar. Infect Dis Poverty. 2017;6 (1):138. doi:10.1186/s40249-017-0353-8

22. Nyunt MH, Aye KM, Kyaw MP, et al. Challenges in universal coverage and utilization of insecticide-treated bed nets in migrant plantation workers in Myanmar. Malar J. 2014;13:211. doi:10.1186/14752875-13-211

23. Manana PN, Kuonza L, Musekiwa A, Mpangane HD, Koekemoer LL. Knowledge, attitudes and practices on malaria transmission in Mamfene, KwaZulu-Natal Province, South Africa 2015. BMC Public Health. 2018;18(1):41. doi:10.1186/s12889-017-4583-2

24. Seck MC, Thwing J, Fall FB, et al. Malaria prevalence, prevention and treatment seeking practices among nomadic pastoralists in northern Senegal. Malar J. 2017;16(1):413. doi:10.1186/s12936-017-2055-X

25. Yukich JO, Taylor C, Eisele TP, et al. Travel history and malaria infection risk in a low-transmission setting in Ethiopia: a case control study. Malar J. 2013;12(1):33. doi:10.1186/1475-2875-12-33

26. Office MH. Metema district annual report; 2017.

27. FDRoE. Federal Democratic Republic of Ethiopia. Ethiopian Population and Housing Census; 2017.

28. Watanabe N, Kaneko A, Yamar S, et al. A prescription for sustaining community engagement in malaria elimination on Aneityum Island, Vanuatu: an application of Health Empowerment Theory. Malar J. 2015;14:291. doi:10.1186/s12936-015-0779-z

29. Arroz JA, Chirrute F, Mendis C, Chande MH, Kollhoff V. Assessment on the ownership and use of mosquito nets in Mozambique. Rev Saude Publica. 2016;50:67. doi:10.1590/s15188787.2016050006335

30. Nguyen TN, Thu PN, Hung NT, et al. Community perceptions of targeted anti-malarial mass drug administrations in two provinces in Vietnam: a quantitative survey. Malar J. 2017;16(1):17. doi:10.1186/ s12936-016-1662-2

31. Matangila JR, Fraeyman J, Kambulu MM, et al. The perception of parents and teachers about intermittent preventive treatment for malaria in school children in a semi-rural area of Kinshasa, in the Democratic Republic of Congo. Malar J. 2017;16(1):19. doi:10.1186/ s12936-016-1670-2
32. World Health Organization. Basic malaria microscopy. Part I: learner's guide. Geneva, Switzerland: World Health Organization; 1991.

33. Cheesbrougu M. District Laboratory Practice in Tropical Countries. 2nd ed. Cambridge University Press; 2006:319-329.

34. Nyirakanani C, Chibvongodze R, Habtu M, Masika M, Mukoko D, Njunwa KJ. Prevalence and risk factors of asymptomatic malaria among underfive children in Huye District, Southern Rwanda. Tanzan J Health Res. 2018;20(1).

35. Berzosa P, de Lucio A, Romay-Barja M, et al. Comparison of three diagnostic methods (microscopy, RDT, and PCR) for the detection of malaria parasites in representative samples from Equatorial Guinea. Malar J. 2018;17(1):333. doi:10.1186/s12936-018-2481-4

36. Maketa V, Mavoko HM, da Luz RI, et al. The relationship between Plasmodium infection, anaemia and nutritional status in asymptomatic children aged under five years living in stable transmission zones in Kinshasa, Democratic Republic of Congo. Malar J. 2015;14 (1):1-9. doi:10.1186/s12936-015-0595-5

37. Kritsiriwuthinan K, Ngrenngarmlert W. Molecular screening of Plasmodium infections among migrant workers in Thailand. J Vector Borne Dis. 2011;48(4):214.

38. Aschale Y, Mengist A, Bitew A, Kassie B, Talie A. Prevalence of malaria and associated risk factors among asymptomatic migrant laborers in West Armachiho District, Northwest Ethiopia. Res Rep Trop Med. 2018;9:95. doi:10.2147/RRTM.S165260

39. Deress T, Girma M. Plasmodium falciparum and Plasmodium vivax Prevalence in Ethiopia: a Systematic Review and Meta-Analysis. Malar Res Treat. 2019;2019:1-12. (Review Article). doi:10.1155/2019/7065064

40. Tadesse F, Fogarty AW, Deressa W. Prevalence and associated risk factors of malaria among adults in East Shewa Zone of Oromia Regional State, Ethiopia: a cross-sectional study. BMC Public Health. 2018;18(1):25. doi:10.1186/s12889-017-4577-0

41. Heinemann M, Phillips RO, Vinnemeier CD, Rolling C, Tannich E, Rolling $\mathrm{T}$ High prevalence of asymptomatic malaria infections in adults, Ashanti Region, Ghana, 2018. 2020.

42. Mvumbi DM, Bobanga TL, Melin P, et al. High prevalence of plasmodium falciparum infection in asymptomatic individuals from the Democratic Republic of the Congo. Malar Res Treat. 2016;2016:1-4. doi:10.1155/2016/5405802

43. Matangila JR, Lufuluabo J, Ibalanky AL, da Luz RAI, Lutumba P, Van Geertruyden J-P. Asymptomatic Plasmodium falciparum infection is associated with anaemia in pregnancy and can be more cost-effectively detected by rapid diagnostic test than by microscopy in Kinshasa, Democratic Republic of the Congo. Malar J. 2014;13 (1):132. doi:10.1186/1475-2875-13-132

44. Abossie A, Yohanes T, Nedu A, Tafesse W, Damitie M. Prevalence of malaria and associated risk factors among Febrile children under five years: a Cross-Sectional Study in Arba Minch Zuria District, South Ethiopia. Infect Drug Resist. 2020;13:363. doi:10.2147/IDR.S223873

45. Institute EPH. Ethiopia National Malaria Indicator Survey. Addis Ababa: EPHI; 2015.

46. Pankti P, Minal T, Nimisha S, Himanshu K. A study of prevalence and seasonal trends of different malarial species in district hospital. Int J Res Med Sci. 2016;4:4155-4157.

47. Suliman MMA, Hamad BM, Albasheer MMA, et al. Molecular evidence of high proportion of plasmodium vivax malaria infection in White Nile Area in Sudan. J Parasitol Res. 2016;2016:1-4. doi:10.1155/2016/2892371

48. Chirebvu E, Chimbari MJ, Ngwenya BN Assessment of risk factors associated with malaria transmission in Tubu Village, Northern Botswana. Hindawi Publishing Corporation. 2014;(Article ID 403069):10.

49. Liu H, Xu J-W, Guo X-R, et al. Coverage, use and maintenance of bed nets and related influence factors in Kachin Special Region II, northeastern Myanmar. Malar J. 2015;14(212):1-12. doi:10.1186/s12936-015-0727-y 
50. Solomon T, Loha E, Deressa W, Gari T, Overgaard HJ, Lidatjorn B. Low use of long-lasting insecticidal nets for malaria prevention in south-central Ethiopia: a community -based cohort study. PLoS One. 2019;10 (1371):1-12.

51. Alemu K, Worku A, Berhane Y, Kumie A. Men Traveling away from home are more likely to bring malaria into high altitude villages, Northwest Ethiopia. PLoS One. 2014;9(4/e95341):1-7. doi:10.1371/ journal.pone.0095341

52. Tefera G. Prevalence of malaria and associated factors among patients attending at Hallaba Health Center, Southern Ethiopia. Immunol Infect Dis. 2014;2(3):25-29.

53. Taylor AR, Watson JA, Chu CS, et al. Resolving the cause of recurrent Plasmodium vivax malaria probabilistically. Nat Commun. 2019;10(1):1-11. doi:10.1038/s41467-019-13412-x

54. White NJ. Determinants of relapse periodicity in Plasmodium vivax malaria. Malar J. 2011;10(1):297.

55. Battle KE, Karhunen MS, Bhatt $\mathrm{S}$, et al. Geographical variation in Plasmodium vivax relapse. Malar J. 2014;13(1):1-16. doi:10.1186/ 1475-2875-13-144

56. Carrasco-Escobar G, Miranda-Alban J, Fernandez-Miñope C, et al. High prevalence of very-low Plasmodium falciparum and Plasmodium vivax parasitaemia carriers in the Peruvian Amazon: insights into local and occupational mobility-related transmission. Malar J. 2017;16(1):415. doi:10.1186/s12936-017-2063-x

57. Vajda E, Webb C. Assessing the risk factors associated with malaria in the highlands of Ethiopia: what do we need to know? Trop Med Infect Dis. 2017;2(1):4. doi:10.3390/tropicalmed2010004

58. Lemma W. Impact of high malaria incidence in seasonal migrant and permanent adult male laborers in mechanized agricultural farms in Metema - humera lowlands on malaria elimination program in Ethiopia. BMC Public Health. 2020;20(1):320. doi:10.1186/s12889-020-8415-4

59. Srivastava HC, Chandrashekar P, Kurien G, Sreehari U, Yadav RS. Malaria in seasonal migrant population in Southern Gujarat, India. Trop Biomed. 2011;28(3):638-645.

60. Nouhoum Diallo PA, Maya E, Aikins M, Sarfo B. Burden of malaria in mobile populations in the Greater Accra region, Ghana; a cross sectional study. Malar J. 2017;16(109):3-9. doi:10.1186/s12936-016-1653-3

61. Nlinwe NO, Ateh TAE. Assessment of malaria predisposing factors among crop production farmers attending the Ndop District Hospital, Northwest Region of Cameroon. J Parasitol Res. 2020;2020:1-8. doi:10.1155/2020/1980709

62. Degarege A, Fennie K, Degarega D, Chennupati S, Madhivana P. Improving Soocioeconomic status may reduce the burden of malaria in sub Saharan Africa: a systematic review and meta-analysis. PLoS One. 2019;14(1):e0211205. doi:10.1371/journal.pone.0211205

63. Mmbando BP, Msangeni HA, Sembuche SH, et al. Epidemiology of malaria in an area prepared for clinical trials in Korogwe, north-eastern Tanzania. Malar J. 2009;8(1):165. doi:10.1186/1475-2875-8-165

64. Tobin-West CI, Kanu EN. Factors influencing the use of malaria prevention methods among women of reproductive age in peri-urban communities of Port harcourt city, Nigeria. Niger Postgrad Med J. 2016;23(1):6-11. doi:10.4103/1117-1936.180114

65. Ayi I, Nonaka D, Adjovu JK, et al. School-based participatory health education for malaria control in Ghana: engaging children as health messengers. Malar J. 2010;9(1):98. doi:10.1186/1475-2875-9-98

66. Krefis AC, Schwarz NG, Nkrumah B, et al. Principal component analysis of socioeconomic factors and their association with malaria in children from the Ashanti Region, Ghana. Malar J. 2010;9(1):201. doi:10.1186/1475-2875-9-201

67. Liu JX, Bousema T, Zelman B, et al. Is housing quality associated with malaria incidence among young children and mosquito vector numbers? Evidence from Korogwe, Tanzania. PLoS One. 2014;9(2): e87358. doi:10.1371/journal.pone. 0087358
68. Snyman K, Mwangwa F, Bigira V, et al. Poor housing construction associated with increased malaria incidence in a cohort of young Ugandan children. Am J Trop Med Hyg. 2015;92(6):1207-1213. doi:10.4269/ajtmh.14-0828

69. Steffanie C, Asnakew KYPB, Worku MW, et al. Malaria control in migrant laborers working in agricultural farms in Metema Region, Ethiopia: current practices, feasibility, and acceptability of new malaria interventions. MACEPA PATH; 2016.

70. Bourdier F, Bunnary C Malaria and population dynam-ics in Cambodia: ethnographic investigations in Païlin, Samlaut and Trapaeng Prasat. IRD Marseille/Phnom Penh; 2010.

71. Wangroongsarb P, Satimai W, Khamsiriwatchara A, et al. Respondent-driven sampling on the Thailand-Cambodia border. II. Knowledge, perception, practice and treatment-seeking behaviour of migrants in malaria endemic zones. Malar J. 2011;10(1):117. doi:10.1186/1475-2875-10-117

72. de Souza TG, Reiners AAO, de Souza Azevedo RC, Fontes CJF, Ferreira RG, Do Carmo PU. Malaria knowledge and treatment adherence in a Brazilian Amazon community. J Infect Dev Ctries. 2016;10 (11):1258-1264. doi:10.3855/jidc.7129

73. Al-Adhroey AH, Nor ZM, Al-Mekhlafi HM, Mahmud R. Opportunities and obstacles to the elimination of malaria from Peninsular Malaysia: knowledge, attitudes and practices on malaria among aboriginal and rural communities. Malar J. 2010;9(1):137. doi:10.1186/1475-2875-9-137

74. Evlampidou I, Danis K, Lenglet A, Tseroni M, Theocharopoulos Y, Panagiotopoulos T. Malaria knowledge, attitudes and practices among migrants from malaria-endemic countries in Evrotas, Laconia, Greece, 2013. Euro Surveill. 2015;20(33):21208. doi:10.2807/1560-7917.es2015.20.33.21208

75. Alelign A, Petros B. Knowledge, attitudes and practices of malaria transmission and preventive measures in Woreta town, Northwest Ethiopia. BMC. 2018;11(491).

76. Aragie TB. Knowledge of malaria prevention and control methods and associated factors among rural households in West Belessa district, North West Ethiopia. Res Square. 2019.

77. Atkinson J-A, Bobogare A, Fitzgerald L, et al. A qualitative study on the acceptability and preference of three types of long-lasting insecticide-treated bed nets in Solomon Islands: implications for malaria elimination. Malar J. 2009;8(1):119. doi:10.1186/1475-2875-8-119

78. Soleimani-Ahmadi M, Vatandoost H, Zare M, Alizadeh A, Salehi M. Community knowledge and practices regarding malaria and long-lasting insecticidal nets during malaria elimination programme in an endemic area in Iran. Malar J. 2014;13(1):1-7. doi:10.1186/1475-2875-13-511

79. Meymandi SK, Forsyth CJ, Soverow J, et al. Prevalence of Chagas Disease in the Latin American-born Population of Los Angeles. Clin Infect Dis. 2017;64(9):1182-1188. doi:10.1093/cid/cix064

80. Ntuku HM, Ruckstuhl L, Julo-Réminiac J-E, et al. Long-lasting insecticidal net (LLIN) ownership, use and cost of implementation after a mass distribution campaign in Kasaï Occidental Province, Democratic Republic of Congo. Malar J. 2017;16(1):22. doi:10.1186/s12936-016-1671-1

81. Lim SS, Fullman N, Stokes A, et al. Net benefits: a multicountry analysis of observational data examining associations between insecticide-treated mosquito nets and health outcomes. Plos Med. 2011;8(9):e1001091. doi:10.1371/journal.pmed.1001091

82. Doda Z, Solomon T, Loha E, Gari T, Lindtjørn B. A qualitative study of use of long-lasting insecticidal nets (LLINs) for intended and unintended purposes in Adami Tullu, East Shewa Zone, Ethiopia. Malar J. 2018;17(1):1-14. doi:10.1186/s12936-018-2209-5

83. Wongsrichanalai C, Barcus MJ, Muth S, Sutamihardja A, Wernsdorfer WH. A review of malaria diagnostic tools: microscopy and rapid diagnostic test (RDT). Am J Trop Med Hyg. 2007;77 (6_Suppl):119-127. doi:10.4269/ajtmh.2007.77.119 


\section{Publish your work in this journal}

Research and Reports in Tropical Medicine is an international, peerreviewed, open access journal publishing original research, case reports, editorials, reviews and commentaries on all areas of tropical medicine, including: Diseases and medicine in tropical regions; Entomology; Epidemiology; Health economics issues; Infectious disease; Laboratory science and new technology in tropical medicine;

Submit your manuscript here: http://www.dovepress.com/research-and-reports-in-tropical-medicine-journal
Parasitology; Public health medicine/health care policy in tropical regions; and Microbiology. The manuscript management system is completely online and includes a very quick and fair peer-review system. Visit http://www.dovepress.com/testimonials.php to read real quotes from published authors. 